\title{
Letter to the Editor: Premature Aging of Fibroblasts from Patients with Duchenne Muscular Dystrophy
}

\author{
RICHARD L. WERTZ AND ALLEN D. ROSES
}

Division of Neurology, Department of Medicine, Duke University Medical Center, Durham, North Carolina, USA

A recent publication by Liechti-Gallati, et al., entitled "Abnormal Growth Kinetics and 5' Nucleotidase Activities in Cultured Skin Fibroblasts from Patients with Duchenne Muscular Dystrophy" claimed that these fibroblasts have altered growth characteristics which represent a premature aging syndrome (4). Their data is in direct conflict with our published findings that show no alterations in the growth kinetics or the in vitro lifespan of fibroblasts from patients with Duchenne muscular dystrophy $(9,10)$. Duchenne muscular dystrophy (DMD) is an X-linked recessive disorder (4), with no clinical indications of premature aging. For this disorder to be a segmental progeroid syndrome, there should consistently be a diminished in vitro replicative lifespan, as is the case with progeria and Werner's syndrome (5). Thus the explanation for the differences in our two sets of data suggests that the diminished response reported by Liechti-Gallati, et al. (4) may not be an intrinsic property of DMD fibroblasts.

In order to examine these differences, we are presenting our data for log-phase growth rate, plating efficiency, and in vitro lifespan (Table 1). The in vitro lifespan data have been published (10). The cell strains used to initiate the cultures were obtained from the mesial aspect of the forearm and in most instances at least one control biopsy was cultured simultaneously with the biopsy from a DMD patient. These cells were cultured in Dulbecco's modification of minimal essential media plus $10 \%$ fetal bovine serum and were passaged at weekly intervals with $0.04 \%$ trypsin buffered with Puck's saline A containing 1 mM EDTA. In several instances we were able to obtain control cultures from normal brothers differing only $1-4$ years in age $\left(A_{3}-A_{4}, A_{33}-A_{34}\right.$, $\left.A_{39}-A_{40}\right)$. The initial outgrowth from the tissue explants was monitored to insure that there were no differences between experimental and control cultures. There were no differences in the log phase growth rate, plating efficiency, or in vitro lifespan (Table 1). Furthermore, we have analyzed the in vitro lifespan from a series of cultures obtained from individuals of differing donor age (Fig. 1) and observed the characteristic inverse relationship between donor age and replicative lifespan first described by Martin, et al. (6). Thus, using methods that reproduce previously published in vitro aging data, we were unable to detect any differences between fibroblast cultures obtained from DMD patients and controls.

The results presented by Liechti-Gallati, et al. (4) appear identical to data obtained when early and late passage cultures are compared (3). The most striking differences reported for DMD fibroblasts were diminished doubling frequency during log phase growth and increased $5^{\prime}$ nucleotidase activity (4). A delay in $\log$ phase doubling time is a common feature of nearly senescent cultures (3). $5^{\prime}$ Nucleotidase has been extensively studied by Sun, et al. (8) in aging systems and the specific activity has been found to increase with age. We have also observed approximately a 4fold increase in specific activity for cells nearing the end of their in vitro lifespan, but no differences were observed between DMD and control cultures (Table 2). Thus it appears that the DMD cell strains examined by Liechti-Gallati, et al., behave in a manner consistent with nearly senescent fibroblasts.
Although Leichti-Gallati, et al. (4) maintain that this must be an intrinsic feature of the fibroblasts obtained from DMD patients, there are other alternative explanations for their data. Harley and Goldstein (2) have documented the extreme variation in the number of population doublings that are possible from cells obtained from the periphery and interior of a monolayer migrating from a tissue explant. Thus, if treatment or sampling of the explant cultures introduced variability, a selectivity bias may have resulted inadvertently. This could lead to a selection of control and DMD cultures that are widely deviant in the number of potential doublings which remain. Liechti-Gallati, et al. (4) also state that all experiments were completed between passage level 5 and 10 , but no information about their passaging regimen is provided. The number of population doublings of the cultures from passage to passage, resulting from differences in growth rate, can result in large differences in the number of population doublings accumulated. This could result in differential aging of the cultures even when passaged under similar conditions. Perhaps the cultures from the DMD patients were seeded at lower density than controls and had exhausted considerably more of their in vitro lifespan. We are unable to calculate this from the data presented.

The data illustrated in Figure 2 of the Liechti-Gallati, et al. (4) paper suggest other possible artifacts. This figure indicates that there was a lag time of at least $72 \mathrm{~h}$ before the cultures entered into log-phase growth. The lag time for healthy early passage fibroblasts is usually only $24 \mathrm{~h}$. In our experience, the only time we have ever observed a lag time as long as $72 \mathrm{~h}$ was when cultures had less than $5 \%$ of the replicative in vitro lifespan remaining. Thus it appears that the cells may have been damaged in some manner. Their reported plating efficiencies were $76.5 \pm 10.74 \%$ (S.E.) for the controls and $78.20 \pm 6.27 \%$ for the DMD cultures. They calculated plating efficiency as the percent cells observed 24 $\mathrm{h}$ after plating $2 \times 10^{3}$ cells $/ \mathrm{cm}^{2}$; however, this only measures cell attachment efficiency, which does not change as sensitively as other parameters used to measure viability and vigor of cultured cells. A far better indicator would have been colony forming ability, which has been shown by Smith, et al. (7) to have a very high degree of correlation with remaining replicative lifespan. This indicates that even though there was cell attachment in their experiments there may have been damage, possibly during trypsinization of the cells. Most laboratories use $0.01-0.05 \%$ trypsin in a buffered saline solution or in basal media. In addition, EDTA is added to avoid clumping of the cells once they are freed from the surface. In the "Materials and Methods" section LiechtiGallati, et al. (4) report that $0.25 \%$ trypsin was used. Neither buffers nor EDTA was mentioned. Furthermore, they incubated the cells in trypsin for 5-10 minutes. It has been our experience that at room temperature $0.04 \%$ trypsin causes irreversible damage when left on the cells for more than $20 \mathrm{~min}$. The primary consequence is slower growth of the cells without any decline in the efficiency of attachment. Because they used 5 times the trypsin concentration, it is quite possible that this could have damaged the cells, possibly in a selective manner. Also if buffers were not 
Table 1. The replicative in vitro lifespan, early passage plating efficiency, and log-phase doubling time of Duchenne muscular dystrophy (DMD) and control cultures

\begin{tabular}{|c|c|c|c|c|}
\hline $\begin{array}{c}\text { Cell } \\
\text { strain }^{1}\end{array}$ & $\begin{array}{c}\text { Donor } \\
\text { age }\end{array}$ & $\begin{array}{c}\text { Plating }^{2} \\
\text { Effi- } \\
\text { ciency }\end{array}$ & $\begin{array}{c}\text { Doubling }^{3} \\
\text { Time (h) }\end{array}$ & $\begin{array}{c}\text { Replication } \\
\text { Life span (CPD) }\end{array}$ \\
\hline \multicolumn{5}{|c|}{ DMD } \\
\hline $\mathrm{A}_{3}$ & 6 & $104 \pm 12.5$ & $27.3 \pm 1.3$ & 62 \\
\hline$A_{10}$ & 8 & $81 \pm 9.5$ & $27.1 \pm 3.8$ & 45 \\
\hline $\mathrm{A}_{33}$ & 13 & $81 \pm 2.2$ & $25.5 \pm 2.4$ & 54 \\
\hline $\mathbf{A}_{39}$ & 11 & $78 \pm 3.3$ & $25.4 \pm 2.8$ & 63 \\
\hline Mean $\pm S . E$ & E. 9.5 & $86 \pm 6.0$ & $26.3 \pm 0.5$ & $56 \pm 4.2$ \\
\hline \multicolumn{5}{|c|}{ Control } \\
\hline $\mathrm{A}_{4}$ & 8 & $83 \pm 9.5$ & $26.9 \pm 2.5$ & 53 \\
\hline$A_{11}$ & 5 & $84 \pm 6.4$ & $22.5 \pm 1.2$ & 54 \\
\hline $\mathrm{A}_{34}$ & 14 & $74 \pm 2.2$ & $20.9 \pm 1.3$ & 70 \\
\hline $\mathrm{A}_{40}$ & 15 & & $24.4 \pm 1.4$ & 55 \\
\hline \multicolumn{2}{|c|}{ Mean \pm S.E. 10.5} & $81 \pm 2.3$ & $23.7 \pm 1.3$ & $58 \pm 4.2$ \\
\hline
\end{tabular}

Statistical comparison DMD versus control

$t$ test $\quad \mathrm{NS}^{5} \quad \mathrm{NS} \quad \mathrm{NS} \quad$ NS

${ }^{1}$ See Wertz, et al. (10) for a description of culture conditions and passaging procedures. Each cell strain was examined in triplicate at passage levels 8-10.

${ }^{2}$ Mean and S.E. for the $\%$ of inoculum remaining $24 \mathrm{~h}$ after plating.

${ }^{3}$ Mean and S.E. of the log phase growth rate from a seeding density of $2.33 \times 10^{3}$ cells $/ \mathrm{cm}^{2}$.

${ }^{4}$ Total cumulated populated doublings (CPD) before onset of senescence.

${ }^{5}$ Not significantly different at $P<0.1$.

Table 2. $5^{\prime}$ Nucleotidase activity in early passage and nearly senescent fibroblast plasma membranes and cell homogenate of Duchenne muscular dystrophy (DMD) and control fibroblasts

\begin{tabular}{|c|c|c|}
\hline & \multicolumn{2}{|c|}{$5^{\prime}$-Nucleotidase activity } \\
\hline & Early passage & Late passage \\
\hline & \multicolumn{2}{|c|}{$\operatorname{DMD}(n=3)$} \\
\hline Plasma membrane & $44.5 \pm 5.2^{1}$ & $210 \pm 16.4$ \\
\hline \multirow{2}{*}{ Cell homogenate } & $15.4 \pm 2.3$ & $56.3 \pm 6.2$ \\
\hline & \multicolumn{2}{|c|}{ Control $(n=3)$} \\
\hline Plasma membrane & $43.6 \pm 7.1$ & $226 \pm 18.2$ \\
\hline $\begin{array}{l}\text { Cell homogenate } \\
t \text { test }\end{array}$ & $\begin{array}{c}14.8 \pm 2.5 \\
\mathrm{NS}^{2}\end{array}$ & $\begin{array}{c}51.2 \pm 4.9 \\
\mathrm{NS}^{2}\end{array}$ \\
\hline
\end{tabular}

${ }^{1}$ Micromoles of inorganic phosphate liberated from $\mathrm{cAMP} / \mathrm{min} / \mathrm{mg}$ total protein.

${ }^{2}$ Not significant at the $P<0.1$ level.

used, there also could be uncontrolled variation in $\mathrm{pH}$ as well. In the "Materials and Methods" section, Liechti-Gallati, et al., describe the use of 14 control and 10 DMD cell strains in their experiments. The results in Table 1 report on varying numbers of strains in each set of data. No criteria were given for selecting the data reported.

Therefore, we believe that several areas of experimental artifact can be identified. The conclusions of this study are of critical importance to investigators interested in the phenotypic expression of genetic mutation in DMD. Thus we feel that it is useful to present and compare the conflicting data and methods.

$\begin{array}{ll}\text { IN VITRO LIFESPAN vs } & Y=-(.40 \pm .12) \times \\ \text { DONOR AGE } & C C=.69 \quad \mathrm{p}<.01 \\ & N=14\end{array}$

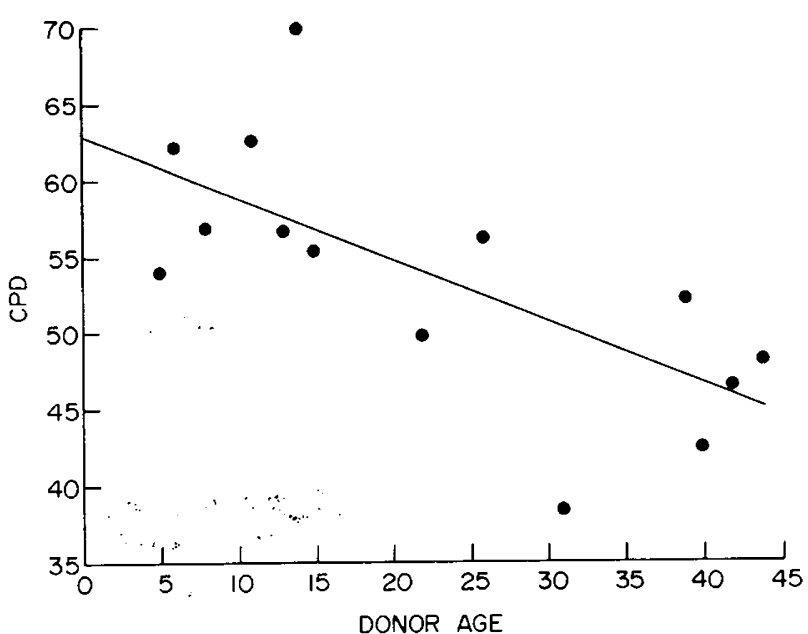

Fig. 1. Inverse relationship between donor age and replicative in vitro lifespan. The human skin fibroblast strains were established and passaged as described in Wertz, et al. (10). These strains were aged in vitro with the constant seeding density method and the cell strains were obtained from normal subjects and patients with myotonic dystrophy or Duchenne muscular dystrophy. The regression coefficient of the line was $y=-(0.40$ $\pm 0.12) \times$, with a y intercept $62.5 \pm 8.3$ cumulated population doublings (CPD) before the onset of senescence. The correlation coefficient was 0.69 (a perfect fit to the line would have been 1) and the probability of these points defining a line with a slope not equal to 0 was $P<0.01$.

\section{REFERENCES AND NOTES}

1. Appel, S. H. and Roses, A. D.: Muscular dystrophies. In: Metabolic Basis of Inherited Disease. (eds.) J. B. Stanbury, J. B. Wyngaarden, and S. Fredrickson. 4th edition. p. 1260 (McGraw-Hill, New York, New York 1978).

2. Harley, C. B. and Goldstein, S.: Cultured human fibroblasts: distribution of cell generations and a critical limit. J. Cell. Physiol., 97: 509 (1978).

3. Hayflick, L.: Recent advances in the cell biology of aging. Mech. Ageing Develop., 14: 59 (1980).

4. Liechti-Gallati, S., Moser, H., Sieqrist, P., Wiesman, U., and Herschkowitz, N. $\mathrm{N}$.: Abnormal growth kinetics and $5^{\prime}$ nucleotidase activities in cultured skin fibroblasts from patients with Duchenne muscular dystrophy. Pediatr. Res., 15: 1411 (1981).

5. Martin, G. H.: Genetic syndromes in man with potential relevance to the pathobiology of aging. In: Genetic Effects on Aging (eds.) D. Bergoma and D. E. Harrison (eds.) p. 5 (Allen R. Liss, New York, New York (1978).

6. Martin, G. M., Sprague, C. A., and Epstein, C. J.: Replicative lifespan of cultivated human cells. Effects of donor's age, tissue and genotype. Lab Invest. 23: $86(1970)$.

7. Smith, J. R., Pereira-Smith, O. M., Braunschweigen, K. I., Roberts, T. W., and Whitney, R. G.: A general method for determining the replicative age of normal animal cell cultures. Mech. Ageing Develop., 12: 355 (1980).

8. Sun, A. S., Alvarez, L. V., Reinach, P. S., and Rubin, E.: $5^{\prime}$ Nucleotidase in normal and virus transformed cells: implications for cellular aging in vitro. Lab. Invest., 41: 1 (1979)

9. Wertz, R. L., Hartwig, G. B., Atwater, S. K., Brophy, J. J., Frost, A. P., and Roses, A. D.: Myotonic dystrophy (a putative progeroid syndrome) and Duchenne dystrophy fibroblasts have normal limits for in vitro lifespan and growth characteristics. J. Cell Biol., 87: 81a (1980).

10. Wertz, R. L., Hartwig, G. B., Frost, A. P., Brophy, J. J., Atwater, S. K., and Roses, A. D.: Patients with myotonic dystrophy, a possible segmental progeroid syndrome, and Duchenne muscular dystrophy have fibroblasts with normal limits for in vitro lifespan and growth characteristics. J. Cell. Physiol., 107: 255 (1981).

11. We thank K. A. Greenleaf for her assistance. This work was supported in part by a Clinical Research Grant from the Muscular Dystrophy Association.

12. Support for Richard $L$. Wertz was provided by an NIH NSRA award $5 T 32$ GM07403 for Biological Membrane Studies. 